\title{
Altitudes of a Tetrahedron and Traceless Quadratic Forms
}

\author{
Hans Havlicek Gunter Weiß
}

1. INTRODUCTION. It is well known that the three altitudes of a triangle are concurrent at the so-called orthocenter of the triangle. So one might expect that the altitudes of a tetrahedron also meet at a point. However, it was already pointed out in 1827 by the Swiss geometer Jakob Steiner (1796-1863) that the altitudes of a general tetrahedron are mutually skew, for they are generators of an equilateral hyperboloid. This is a hyperboloid with the following rather peculiar property: each nontangential plane that is perpendicular to a generator meets the hyperboloid along an equilateral hyperbola, i.e., a hyperbola with orthogonal asymptotes.

There are many papers, especially from the nineteenth century, that deal with the altitudes of tetrahedra and related topics. An excellent survey article with many historical footnotes is the paper of N.A. Court [7. Another major source is the article of M. Zacharias in the Encyclopedia of Mathematical Sciences [26] (completed in 1913). Also, the book of H. Schröter [24] contains in section 28 some interesting remarks on older papers that are not cited elsewhere.

Some of the many special points and lines associated with triangles extend to tetrahedra, others do not. More precisely, there are often a number of possibilities for generalizing a concept (like the orthocenter) from the plane to 3-space (or even $n$-space), since separate notions in higher-dimensional geometry may coincide in the plane. We illustrate this in section 3 , where we discuss the Monge point of a tetrahedron. If the definition of the Monge point is applied directly to the planar case, then the orthocenter of a triangle is obtained. Table 1 starts with some well-known "noteworthy points" of a triangle and lists their existence in higher dimensions, together with short remarks or references.

\begin{tabular}{|c|c|c|c|}
\hline Dimension & $n=2$ & $n=3$ & $n \geq 4$ \\
\hline Object & Triangle & Tetrahedron & $n$-Simplex \\
\hline Centroid $G$ & Yes & Yes & Yes (See [20], [22].) \\
\hline Circumcenter $C$ & Yes & Yes & Yes (See $[20,[22]$.) \\
\hline Incenter $I$ & Yes & Yes & Yes (See [20], [22].) \\
\hline Orthocenter $H$ & Yes & $\begin{array}{l}\text { Sometimes } \\
(\text { sec. } 2)\end{array}$ & $\begin{array}{c}\text { Sometimes } \\
(\text { See [17, [20].) }\end{array}$ \\
\hline Monge point $M$ & $\begin{array}{l}\text { Yes: } M=H \\
\quad \text { (sec. } 3 \text { ) }\end{array}$ & Yes (sec. 3 & Yes (See [17, [20].) \\
\hline $\begin{array}{l}\text { Euler line } e \\
(G, C, M \in e)\end{array}$ & $\begin{array}{c}\text { Yes } \\
(G, C, H \in e)\end{array}$ & Yes (sec. 4 ) & Yes (See [17, [20].) \\
\hline
\end{tabular}

Table 1.

Clearly, this list is far from being complete. For example, Feuerbach's famous 9 -point circle for the triangle is generalized in [10, to the $n$-dimensional case as a 
Feuerbach hypersphere, and in 7 two 12-point spheres for an orthocentric tetrahedron are described. However, in higher dimensions many questions are still open and have not been treated systematically. Further references are given in section 7 .

There are two major reasons for revisiting the subject "altitudes of a tetrahedron." On the one hand, we want to visualize the results, since there are hardly any pictures in the cited papers. The figures in this article have been prepared with the computer algebra system Maple. Clearly, a figure must not replace a formal proof, but we are convinced that figures can assist the reader in better understanding spatial relationships. Also, in certain instances the idea behind a proof is rather immediate from an adequate illustration.

On the other hand, we aim at a modern coordinate-free presentation in terms of analytic geometry based on a Euclidean vector space, whereas a lot of papers on the subject are written in terms of synthetic projective geometry. Thus the prerequisite for reading this article is knowledge of basic linear algebra.

We shall see that the quadric surface carrying the altitudes of a general tetrahedron appears as a level set of a traceless (trace $=0$ ) quadratic form $Q^{*}$. In fact, there is a natural link between tetrahedra and certain traceless quadratic forms that will lead us to an explicit expression for $Q^{*}$.

2. ALTITUDES AND ORTHOCENTRIC PERPENDICULARS. Let $\left\{A_{0}, A_{1}, A_{2}, A_{3}\right\}$ be the set of vertices of a nondegenerate tetrahedron $T$ in Euclidean 3 -space, i.e., assume that these four points are not coplanar. Throughout this paper it is tacitly assumed that $i, j, k$, and $l$ are indices subject to the requirement that $\{i, j, k, l\}=\{0,1,2,3\}$. So, if $l$ is the index of the "top vertex," then $i, j$, and $k$ are the indices of the "base." Since we are going to describe points by vectors of an underlying Euclidean vector space $\mathbb{E}$, we have to fix an origin $O$. For the moment $O$ is chosen arbitrarily, although in section 6 we make a specific choice of origin in order to simplify our calculations.

Each vertex $A_{i}$ is given by its position vector $a_{i}:=\overrightarrow{O A_{i}}$. Also, we introduce the vectors

$$
b_{i j}:=a_{i}-a_{j} .
$$

These vectors have the following basic properties:

$$
\begin{gathered}
b_{i j}+b_{j i}=0 \\
b_{i j}+b_{j k}+b_{k i}=0 \\
b_{i j}+b_{j k}+b_{k l}+b_{l i}=0 \\
b_{i j}, b_{i k}, b_{i l} \text { are linearly independent. }
\end{gathered}
$$

Observe that (4) follows from our assumption that $T$ is nondegenerate. The definition (1) and properties of the dot product in $\mathbb{E}$ give the identity

$$
b_{i j} \cdot b_{k l}+b_{i k} \cdot b_{l j}+b_{i l} \cdot b_{j k}=0 .
$$

Its geometric meaning will be explained in due course.

We consider an edge $A_{i} A_{j}$ of $T$ and one of the remaining two vertices, say $A_{k}$. The plane through $A_{k}$ perpendicular to the specified edge is the set of all points $X$ described by the equation

$$
b_{i j} \cdot\left(a_{k}-x\right)=0,
$$

where $x:=\overrightarrow{O X}$ denotes an "unknown vector" in $\mathbb{E}$. We observe that there are at most twelve such planes. They will be the key to many of our considerations. 
We write $h_{l}$ for the altitude of $T$ passing through $A_{l}$. It lies in every plane through $A_{l}$ that is perpendicular to the opposite face. Hence $h_{l}$ lies in the three planes with equations

$$
\begin{aligned}
& b_{i j} \cdot\left(a_{l}-x\right)=0, \\
& b_{j k} \cdot\left(a_{l}-x\right)=0, \\
& b_{k i} \cdot\left(a_{l}-x\right)=0 .
\end{aligned}
$$

It follows from (4) and (2) that any two of these equations are linearly independent, whereas (3) implies that all three equations are linearly dependent. Thus (7) describes three different planes that meet at the altitude $h_{l}$, any two of which are already sufficient to determine $h_{l}$. Figure 1 shows those planes through the altitude $h_{3}$ that are perpendicular to $A_{0} A_{1}$ and $A_{0} A_{2}$, respectively.

We now consider those uniquely determined planes through $A_{k}, A_{i}$, and $A_{j}$ that are parallel to the first, the second, and the third plane of (7), respectively. This yields the linear system

$$
\begin{aligned}
& b_{i j} \cdot\left(a_{k}-x\right)=0, \\
& b_{j k} \cdot\left(a_{i}-x\right)=0, \\
& b_{k i} \cdot\left(a_{j}-x\right)=0 .
\end{aligned}
$$

Again, this system describes three planes that intersect in a line, since

$$
b_{i j} \cdot a_{k}+b_{j k} \cdot a_{i}+b_{k i} \cdot a_{j}=0
$$

follows from (1), and $\left(b_{i j}+b_{j k}+b_{k i}\right) \cdot x=0 \cdot x=0$ is immediate from (3). Let us write $n_{l}$ for the line given by (8). (Two of these planes through $n_{3}$ are drawn in Figure 1.) From its definition, the line $n_{l}$ contains the orthocenter of the triangle with vertices $A_{i}, A_{j}$, and $A_{k}$. Also, it is perpendicular to the plane of that triangle. We call $n_{l}$ an orthocentric perpendicular of $T$.

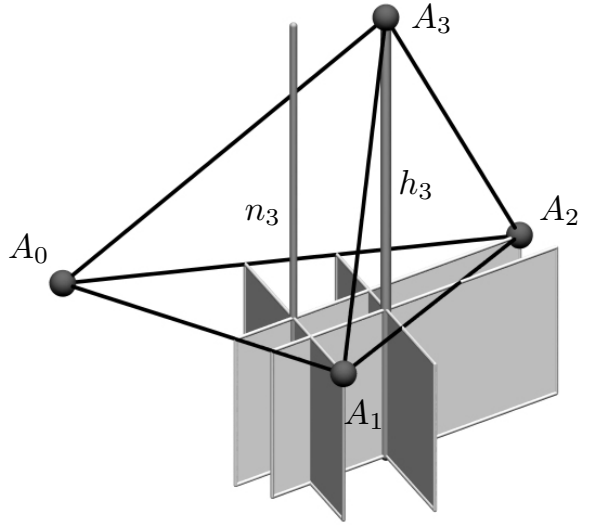

Figure 1 .

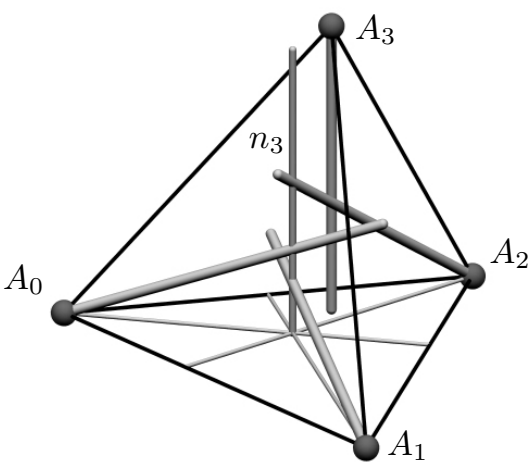

Figure 2

The altitude $h_{l}$ and the perpendicular $n_{l}$ are parallel, but in general they are not the same. However, we have the following result.

Theorem 1. Each orthocentric perpendicular $n_{l}$ meets every altitude $h_{i}$ with $i \neq l$.

Proof. We read off from the second equation in (7) (after interchanging $i$ with $l$ ) and the second equation in (8) that $h_{i}$ and $n_{l}$ are both contained in the plane with 
equation $b_{j k} \cdot\left(a_{i}-x\right)=0$. Furthermore, because $h_{i}$ and $n_{l}$ are orthogonal to different faces of the tetrahedron $T$, they cannot be parallel. As a result, the two lines have a point in common.

In Figure 2 the line $n_{3}$ meets three out of the four altitudes of $T$. Also, under orthogonal projection onto the plane $A_{0} A_{1} A_{2}$ the line $n_{3}$ is mapped to the orthocenter of the triangle with vertices $A_{0}, A_{1}, A_{2}$, and the altitudes $h_{0}, h_{1}$, and $h_{2}$ project to the altitudes of that triangle. We can see that the foot of $h_{3}$ is on no altitude of the opposite triangle. Hence $h_{3}$ does not meet any other altitude of the tetrahedron. So we can seek a criterion for deciding whether or not two altitudes meet at a point. It will be convenient to say that two lines (with or without a common point) are orthogonal if their direction vectors are orthogonal.

Theorem 2. An altitude $h_{i}$ meets an altitude $h_{j}$ precisely when

$$
b_{k l} \cdot b_{i j}=0
$$

or, in other words, when the opposite edges $A_{k} A_{l}$ and $A_{i} A_{j}$ are orthogonal.

Proof. From (7), the altitude $h_{i}$ is described by the linear system

$$
\begin{aligned}
& b_{j k} \cdot\left(a_{i}-x\right)=0, \\
& b_{k l} \cdot\left(a_{i}-x\right)=0,
\end{aligned}
$$

whereas $h_{j}$ is determined by

$$
\begin{aligned}
& b_{k l} \cdot\left(a_{j}-x\right)=0 \\
& b_{l i} \cdot\left(a_{j}-x\right)=0 .
\end{aligned}
$$

Now (10) implies that $b_{k l} \cdot a_{i}=b_{k l} \cdot a_{j}$. So two of these four equations are the same. Hence $h_{i} \cap h_{j} \neq \emptyset$, for by (4) the three planes given by those equations are not parallel to a line.

Conversely, let $P$ be a common point of $h_{i}$ and $h_{j}$. Put $p=\overrightarrow{O P}$. We infer from

$$
0=0-0=b_{k l} \cdot\left(a_{i}-p\right)-b_{k l} \cdot\left(a_{j}-p\right)=b_{k l} \cdot b_{i j}
$$

that 10$)$ is satisfied.

By symmetry, condition 10 is also necessary and sufficient for the altitudes $h_{k}$ and $h_{l}$ to have a point in common. Hence, if two altitudes intersect, then so do the other two. Moreover, the reader will easily verify that $\sqrt{10}, n_{i} \cap n_{j} \neq \emptyset$, and $n_{k} \cap n_{l} \neq \emptyset$ are mutually equivalent.

At a first glance the following result may be somewhat surprising:

Theorem 3. If one altitude meets two other altitudes then all altitudes are concurrent.

Proof. Suppose that $h_{i}$ meets $h_{j}$ and $h_{k}$. Then 10 implies $b_{k l} \cdot b_{i j}=b_{j l} \cdot b_{i k}=0$ and (5) shows that $b_{i l} \cdot b_{j k}=0$, i.e., $h_{i}$ meets also $h_{l}$. By Theorem 2 , any two altitudes intersect. Clearly, the four altitudes are not coplanar. So they have a common point. 
A tetrahedron with exactly one pair of opposite orthogonal edges is called semiorthocentric. Figure 3 shows such a tetrahedron $\left(A_{0} A_{3} \perp A_{1} A_{2}\right)$. Observe that the foot of $h_{3}$ is on exactly one altitude of the opposite triangle. In Figure 4 , however, all four altitudes are concurrent at a point. Such a tetrahedron is said to be orthocentric, the orthocenter being the point where the altitudes meet. In an orthocentric tetrahedron every edge is orthogonal to its opposite edge. Furthermore, each altitude $h_{i}$ coincides with the orthocentric perpendicular $n_{i}$.

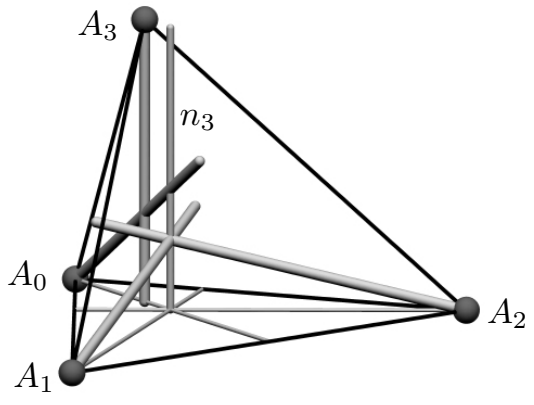

Figure 3 .

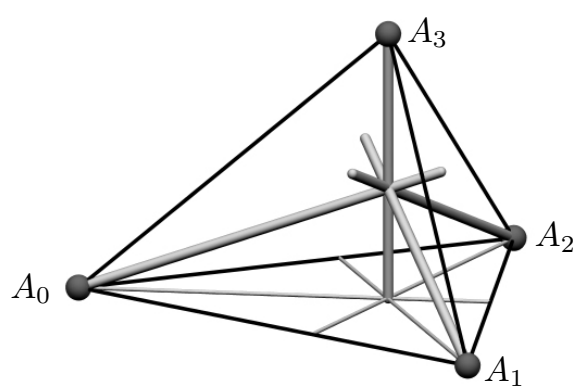

Figure 4

We refer to [1, p. 371], 6], and [9] for other proofs of Theorems 1, 2, and 3

3. THE MONGE POINT OF A TETRAHEDRON. For an orthocentric tetrahedron the intersection of all planes given by (6) is its orthocenter. However, we focus on an arbitrary tetrahedron $T$. Then among the planes (6) there are two that are perpendicular to $A_{i} A_{j}$ and pass through $A_{k}$ and $A_{l}$, respectively. These two planes are either identical or disjoint. (In Figure 1 two such pairs of parallel planes can be seen.) In either case their midplane is the plane with equation

$$
b_{i j} \cdot\left(a_{k}+a_{l}-2 x\right)=0 .
$$

This midplane is orthogonal to the edge $A_{i} A_{j}$, and it passes through the midpoint of the opposite edge $A_{k} A_{l}$ rather than the midpoint of the edge $A_{i} A_{j}$, as the perpendicular bisector of $A_{i} A_{j}$ does. In general, the midplane (11) is therfore not the perpendicular bisector of the edge $A_{i} A_{j}$. The equation of that plane is given in equation (13). The tetrahedron $T$ has six midplanes.

Now for an arbitrary tetrahedron $T$ there will be no orthocenter, but $T$ will have a point discovered by Gaspard Monge (1746-1818) that is now called its Monge point. The construction of this point goes as follows:

Theorem 4. All six midplanes of a tetrahedron are concurrent at a point.

Proof. From (4) we know that the vectors $b_{01}, b_{02}$, and $b_{03}$ are linearly independent. Hence there exists a unique common point, say $M$, of the (mutually nonparallel) planes whose equations are

$$
\begin{aligned}
& b_{01} \cdot\left(a_{2}+a_{3}-2 x\right)=0 \\
& b_{02} \cdot\left(a_{1}+a_{3}-2 x\right)=0 \\
& b_{03} \cdot\left(a_{1}+a_{2}-2 x\right)=0 .
\end{aligned}
$$


We subtract the first equation from the second and obtain

$$
\begin{aligned}
0 & =b_{02} \cdot a_{1}-b_{01} \cdot a_{2}+\left(b_{02}-b_{01}\right) \cdot\left(a_{3}-2 x\right) \\
& =b_{02} \cdot a_{1}+b_{10} \cdot a_{2}+b_{12} \cdot\left(a_{3}-2 x\right) \\
& =b_{12} \cdot\left(a_{0}+a_{3}-2 x\right)
\end{aligned}
$$

where we have used equations (2), (3), and (9). So $M$ lies in the midplane that is perpendicular to the edge $A_{1} A_{2}$. A similar calculation shows that $M$ belongs to the remaining midplanes as well.

Figure 5 displays a tetrahedron and its Monge point $M$. It lies on that line of the plane spanned by the parallel lines $h_{i}$ and $n_{i}$ which is equidistant from both; one such line (dotted) is illustrated for $i=3$. If we deform $T$ by "sliding" the vertex $A_{3}$ down along the line $h_{3}$, holding all other vertices fixed, then the center of gravity of $T$ remains an inner point of $T$, while the circumcenter of $T$ approaches "negative infinity," since the radius of the circumscribed sphere of $T$ tends to infinity. We shall see in section 4 that the center of gravity of $T$ is the midpoint of $M$ and the circumcenter of $T$. Hence the Monge point $M$ "moves up" along the dotted line. This gives Figure 6, in which the Monge point is exterior to the tetrahedron. For the proper choice of $A_{3}$ we could also produce a tetrahedron whose the Monge point is incident with a face.

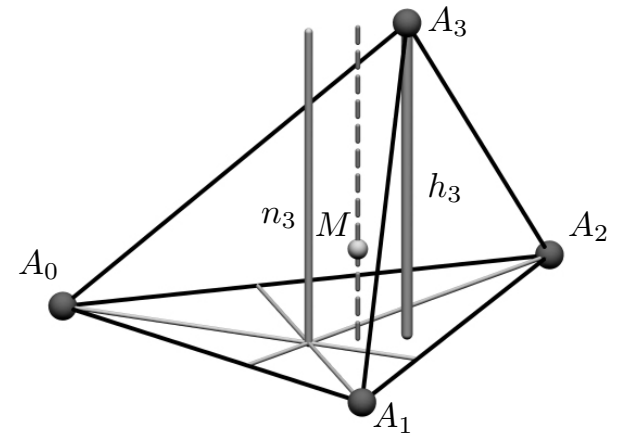

Figure 5 .

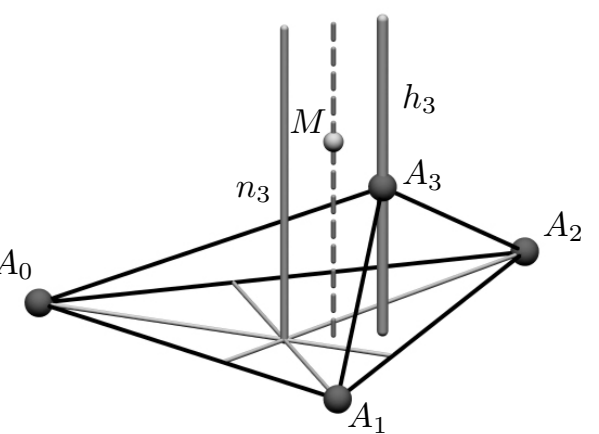

Figure 6

Let $m:=\overrightarrow{O M}$ be the position vector of the Monge point $M$ described in Theorem 4. Then (11) implies that $b_{i j} \cdot\left(a_{k}+a_{l}-2 m\right)=b_{k l} \cdot\left(a_{i}+a_{j}-2 m\right)$ and multiplying this out leads to the relation

$$
2 a_{i} \cdot a_{l}-2 a_{i} \cdot m-2 a_{l} \cdot m=2 a_{j} \cdot a_{k}-2 a_{k} \cdot m-2 a_{j} \cdot m
$$

which is equivalent to

$$
\left(a_{i}-m\right) \cdot\left(a_{l}-m\right)=\left(a_{j}-m\right) \cdot\left(a_{k}-m\right) .
$$

This pretty equation illustrates in another way the special role of the point $M$.

Clearly, if all altitudes of $T$ are concurrent, then the Monge point $M$ is their point of intersection. This is one rationale for the assertion that the Monge point serves as a substitute for the "missing orthocenter" of a general tetrahedron.

We sketch the definition of the Monge point in a more general context in order to illustrate that the orthocenter of a triangle can be considered as its Monge point. An $n$-simplex $S$ in $n$-dimensional Euclidean space has $n+1$ vertices. For each edge there is a unique hyperplane that is perpendicular to that edge and that contains the 
center of gravity of the $n-1$ "opposite vertices," i.e., the vertices not on the given edge. There are $\left(\begin{array}{c}n+1 \\ 2\end{array}\right)$ such hyperplanes, and they have a point in common-the Monge point of the $n$-simplex (see [17 or [20]). For $n=3$ (tetrahedron) this is in accordance with Theorem 44, since each edge has two opposite vertices whose center of gravity is just their midpoint. For $n=2$ (triangle) each edge has a single opposite vertex that is its own center of gravity. In this situation, the three altitudes of the triangle replace the hyperplanes from the general case, and their common point (the orthocenter) coincides with the Monge point.

4. THE EULER LINE IN SPACE. We consider the circumcenter $C$, the center of gravity $G$, and the Monge point $M$ of the tetrahedron $T$ with vertex set $\left\{A_{0}, A_{1}, A_{2}, A_{3}\right\}$. An equation

$$
b_{i j} \cdot\left(a_{i}+a_{j}-2 x\right)=0
$$

describes the perpendicular bisector of the edge $A_{i} A_{j}$, so that $\overrightarrow{O C}$ is the only solution of the linear system of all six equations 13 .

If we fix indices $i$ and $j$, then the midplane of the planes represented by (11) and (13) has an equation of the form

$$
b_{i j} \cdot\left(a_{i}+a_{j}+a_{k}+a_{l}-4 x\right)=0 .
$$

This plane contains the midpoint of the segment $C M$. From (4) we know that there are three linearly independent vectors among the $b_{i j}$, whence the only solution of the linear system comprising all equations 14 is

$$
\overrightarrow{O G}=\frac{1}{4}\left(a_{i}+a_{j}+a_{k}+a_{l}\right) .
$$

This means that the center of gravity is the midpoint of the segment $C M$. In other words, whenever two of the points $G, C$, and $M$ are different, their join can be considered as an analog of the Euler line in 3-space. (We remind the reader that the Euler line of a triangle contains the center of gravity, the circumcenter, and orthocenter of the given triangle.)

We add in passing that in the $n$-dimensional setting there is also an Euler line: the center of gravity divides the segment formed by the circumcenter and the Monge point internally in the ratio $2:(n-1)$ (see [17 or [20). This explains why in the plane (orthocenter $=$ Monge point) the ratio on the Euler line is $2: 1$, whereas in three dimensions it is $2: 2$.

5. TRACELESS QUADRATIC FORMS. If $Q: \mathbb{E} \rightarrow \mathbb{R}$ is a nonzero quadratic form and $\rho$ in $\mathbb{R}$ is a constant, then $Q(x)=\rho$ is an equation of a (possibly degenerate) quadric surface that is symmetric with respect to the origin $O$. We refer to 12 for basic properties of quadrics and quadratic forms.

By the polarization formula, a quadratic form $Q: \mathbb{E} \rightarrow \mathbb{R}$ gives rise to a symmetric bilinear form $f: \mathbb{E} \times \mathbb{E} \rightarrow \mathbb{R}$,

$$
f(v, w)=\frac{1}{2}(Q(v+w)-Q(v)-Q(w)),
$$

with the property $Q(v)=f(v, v)$ for all vectors $v$ in $\mathbb{E}$. This function $f$ is called the polar form of $Q$. 
Let $\left\{e_{1}, e_{2}, e_{3}\right\}$ be an orthonormal basis of $\mathbb{E}$. Then each vector $x$ is uniquely determined by its Cartesian coordinates $\left(\xi_{1}, \xi_{2}, \xi_{3}\right)$ in $\mathbb{R}^{3}$, viz., $x=\xi_{1} e_{1}+\xi_{2} e_{2}+\xi_{3} e_{3}$. The symmetric 3-by-3 matrix $\Sigma=\left(\sigma_{r s}\right):=\left(f\left(e_{r}, e_{s}\right)\right)$ allows us to express $Q$ in terms of coordinates in the form

$$
Q(x)=\sum_{r, s=1}^{3} \sigma_{r s} \xi_{r} \xi_{s} .
$$

If we change to another orthonormal basis, then $\Sigma$ changes to a congruent matrix $\Sigma^{\prime}=\Omega^{\mathrm{T}} \Sigma \Omega$, where $\Omega$ is an orthogonal matrix and $\Omega^{\mathrm{T}}$ denotes the transpose of $\Omega$. As $\Omega$ is orthogonal, we have $\Omega^{\mathrm{T}}=\Omega^{-1}$. The matrices $\Sigma$ and $\Sigma^{\prime}$ are thus similar. It is well known that similar matrices have the same trace. So it makes sense to speak of the trace $\operatorname{tr} Q$ of a quadratic form $Q$, as long as we restrict ourselves to orthonormal bases.

Consider, for example, arbitrary vectors $c$ and $d$ of $\mathbb{E}$ with Cartesian coordinates $\left(\gamma_{1}, \gamma_{2}, \gamma_{3}\right)$ and $\left(\delta_{1}, \delta_{2}, \delta_{3}\right)$, respectively, and the quadratic form

$$
x \mapsto(x \cdot c)(x \cdot d) .
$$

The $(r, s)$-entry of its matrix equals $\frac{1}{2}\left(\gamma_{r} \delta_{s}+\gamma_{s} \delta_{r}\right)$, whence the trace of 15 is

$$
\gamma_{1} \delta_{1}+\gamma_{2} \delta_{2}+\gamma_{3} \delta_{3}=c \cdot d \text {. }
$$

We are particularly interested in quadratic forms with trace zero or, in other words, traceless quadratic forms. If $Q$ is an arbitrary quadratic form on $\mathbb{E}$ then, by transformation on principal axes, there exists an orthonormal basis $\left\{e_{1}, e_{2}, e_{3}\right\}$ of $\mathbb{E}$ with respect to which

$$
Q(x)=\sigma_{11} \xi_{1}^{2}+\sigma_{22} \xi_{2}^{2}+\sigma_{33} \xi_{3}^{2} .
$$

Suppose now that $\operatorname{tr} Q=0$. Hence

$$
\operatorname{tr} Q=\sigma_{11}+\sigma_{22}+\sigma_{33}=0
$$

and there are several cases to consider. These depend on the rank of $Q$, meaning the rank of any associated matrix.

Case 1: $\operatorname{rank} Q \leq 1$. Then at most one coefficient $\sigma_{r r}$ is nonzero and 18 shows that $\sigma_{11}=\sigma_{22}=\sigma_{33}=0$, i.e., $Q$ is the zero-form.

Case 2: $\operatorname{rank} Q=2$. Then the basis can be chosen in such a way that $\sigma_{33}=0$. This means that $\sigma_{22}=-\sigma_{11} \neq 0$ and $Q(x)=\sigma_{11}\left(\xi_{1}-\xi_{2}\right)\left(\xi_{1}+\xi_{2}\right)$. Consequently, $Q(x)=0$ describes a pair of orthogonal planes with equations $\xi_{1}=\xi_{2}$ and $\xi_{1}=-\xi_{2}$, respectively.

Case 3: $\operatorname{rank} Q=3$. Then $Q$ is indefinite by $(18)$ and $Q(x)=0$ is the equation of a quadratic cone (with vertex at the origin) that is called an equilateral cone. Its generators (i.e., the lines entirely contained in the cone) have the following remarkable property (see [25, p. 293, Ex. 19]):

Theorem 5. If $g$ is a generator of an equilateral cone, then there are generators $g_{1}$ and $g_{2}$ of the cone such that $g, g_{1}$, and $g_{2}$ are mutually orthogonal.

Proof. As earlier, let the cone be given in the form $Q(x)=0$, where $Q$ is a traceless quadratic form of rank 3 . Choose $e_{3}$ to be a unit vector in the direction of the generator $g$. Also, let $\left\{e_{1}, e_{2}\right\}$ be an orthonormal basis of $g^{\perp}$ (the orthogonal complement of 
$g$ ) giving the principal axes of $Q$ restricted to $g^{\perp}$. Then the matrix of the associated polar form $f$ reads

$$
\left(\begin{array}{ccc}
\sigma_{11} & 0 & \sigma_{13} \\
0 & \sigma_{22} & \sigma_{23} \\
\sigma_{31} & \sigma_{32} & 0
\end{array}\right)
$$

and $\operatorname{rank} Q=3$ implies that $\sigma_{11}=-\sigma_{22} \neq 0$. We infer that the plane $g^{\perp}$ meets the cone in the orthogonal lines $g_{1}:=\mathbb{R}\left(e_{1}+e_{2}\right)$ and $g_{2}:=\mathbb{R}\left(e_{1}-e_{2}\right)$.

We conclude that an equilateral cone carries infinitely many orthogonal tripods. Figure 7 shows an equilateral cone, some of its orthogonal tripods (one in white, the others in black), and the principal axes.

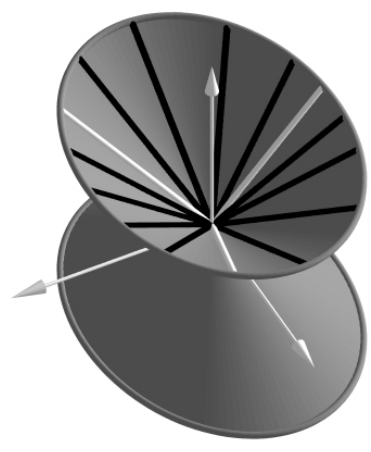

Figure 7

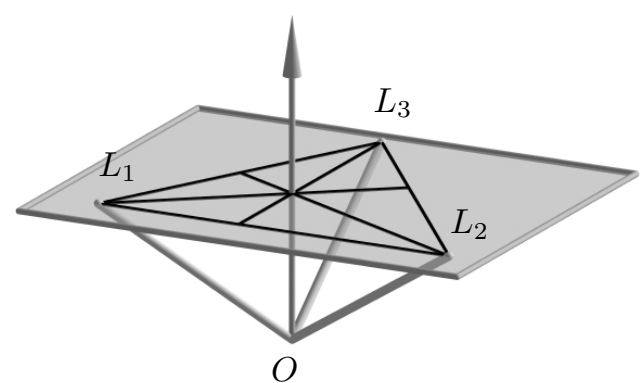

Figure 8

Theorem 5 can be transferred to a result in the plane as follows: Choose any orthogonal tripod on the cone and a plane that meets the legs of the tripod in distinct points, say $L_{1}, L_{2}$, and $L_{3}$ (see Figure 8). Hence $\left\{O, L_{1}, L_{2}, L_{3}\right\}$ determines a so-called trirectangular tetrahedron. The edges $O L_{1}, O L_{2}$, and $O L_{3}$ are at the same time altitudes of this tetrahedron, and $O$ is its orthocenter. Therefore the orthocentric perpendicular of the triangle $\Delta$ with vertices $L_{1}, L_{2}$, and $L_{3}$ runs through $O$ or, said differently, the orthogonal projection of $O$ onto the plane $L_{1} L_{2} L_{3}$ is just the orthocenter of this triangle. It is also worth noting that $\Delta$ is always an acute triangle. Consider, for example, the angle $\varphi_{1}=\angle L_{2} L_{1} L_{3}$. From the law of Pythagoras we get

$$
{\overline{L_{1} L_{2}}}^{2}+{\overline{L_{1} L_{3}}}^{2}={\overline{O L_{1}}}^{2}+{\overline{O L_{2}}}^{2}+{\overline{O L_{1}}}^{2}+{\overline{O L_{3}}}^{2}={\overline{L_{2} L_{3}}}^{2}+2{\overline{O L_{1}}}^{2} .
$$

Now the cosine law, applied to $\Delta$ and $\varphi_{1}$, implies that

$$
2{\overline{O L_{1}}}^{2}=2 \overline{L_{1} L_{2}} \overline{L_{1} L_{3}} \cos \varphi_{1} .
$$

Since $\overline{O L_{1}}, \overline{L_{1} L_{2}}$, and $\overline{L_{1} L_{3}}$ are positive real numbers, so is $\cos \varphi_{1}$, i.e., we have an acute angle at $L_{1}$.

Let us turn back to the orthogonal tripods of an equilateral cone $C$ with an equation $Q(x)=0$ given in coordinates according to $(17)$ and (18); we may assume $\sigma_{11}, \sigma_{22}>0$ and $\sigma_{33}<0$. We select a plane $P: \xi_{3}=\rho$, where $\rho \neq 0$. Then $P$ meets $C$ along an ellipse $E$. This gives Figure 9 the infinitely many orthogonal tripods on the cone give rise to an infinite family of acute triangles that are inscribed in $E$. Furthermore, the center of $E$ is the common orthocenter of all these triangles. Clearly, if the ellipse happens to be a circle $\left(\sigma_{11}=\sigma_{22}\right)$, then all triangles will be equilateral. 


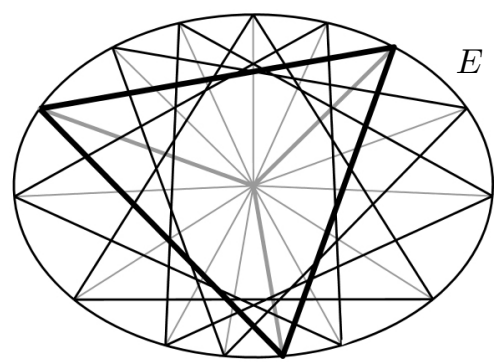

Figure 9 .

Conversely, it is easily seen that every ellipse arises in this way as a planar section of an equilateral cone. Hence the problem of finding all triangles with the aforementioned properties always has infinitely many solutions. Such a problem is called a porism. The investigation of problems of this kind goes back to the French geometer J.V. Poncelet (1788-1867), and it is best understood in terms of projective geometry: the triangles under consideration are not only inscribed in the given ellipse but are also self-polar with respect to an elliptic polarity. It follows that the sides of the triangles are the tangents to another ellipse. The interested reader should consult [25, chaps. 4, 5].

6. THE QUADRIC OF THE ALTITUDES. In this section we choose the Monge point $M$ of a given tetrahedron $T$ as the origin. This will simplify our calculations. Furthermore, we introduce the scalars

$$
\lambda_{i j}:=a_{i} \cdot a_{j}(i \neq j) .
$$

From the symmetry of the dot product we have $\lambda_{i j}=\lambda_{j i}$, but it is more important to notice that from $m=\overrightarrow{M M}=0$ and $\sqrt{12}$ follows

$$
\lambda_{i l}=\lambda_{j k}
$$

i.e., each $\lambda_{i j}$ is equal to one of the numbers $\lambda_{01}, \lambda_{02}$, or $\lambda_{03}$.

First, let us rewrite the condition from (10) from Theorem 2. We see that the altitudes $h_{i}$ and $h_{j}$ meet at a point precisely when

$$
b_{k l} \cdot b_{i j}=\left(a_{k}-a_{l}\right) \cdot\left(a_{i}-a_{j}\right)=2\left(\lambda_{k i}-\lambda_{l i}\right)=2\left(\lambda_{l j}-\lambda_{k j}\right)=0 .
$$

Thus for a "generic" tetrahedron the scalars $\lambda_{01}, \lambda_{02}$, and $\lambda_{03}$ are distinct, for a semiorthocentric tetrahedron exactly two of them are identical, and for a tetrahedron to be orthocentric it is necessary and sufficient that $\lambda_{01}=\lambda_{02}=\lambda_{03}$.

Next, we look for quadratic forms on $\mathbb{E}$ that arise from $T$ in a natural way. We start with the forms

$$
Q_{i j k l}: x \mapsto\left(x \cdot b_{i j}\right)\left(x \cdot b_{k l}\right) .
$$

Since $M$ has been chosen as the origin, every quadric $Q_{i j k l}(x)=0$ is the union of two midplanes, namely, the planes with equations $b_{i j} \cdot x=0$ and $b_{k l} \cdot x=0$ (cf. (11)).

The collection of all quadratic forms $\mathbb{E} \rightarrow \mathbb{R}$ constitutes a real vector space $\mathcal{Q}$ isomorphic to the six-dimensional space of symmetric 3-by-3 matrices over $\mathbb{R}$. However, we are only interested in certain subspaces of $\mathcal{Q}$ :

Theorem 6. The subspace $\mathcal{S}$ of $\mathcal{Q}$ that is spanned by all quadratic forms $Q_{i j k l}$ has dimension two. The subspace $\mathcal{T}$ of all traceless quadratic forms in $\mathcal{S}$ is either oneor two-dimensional. 
Proof. By (2), $Q_{i j k l}=Q_{k l i j}=-Q_{j i k l}$. So each $Q_{i j k l}$ is equal to $\pm Q_{0123}, \pm Q_{0231}$, or $\pm Q_{0312}$. From (1) and a straightforward calculation it follows that

$$
Q_{0123}(x)+Q_{0231}(x)+Q_{0312}(x)=0
$$

for all $x$ in $\mathbb{E}$. We infer that $\mathcal{S}$ is generated by $Q_{0123}$ and $Q_{0231}$. The midplanes perpendicular to $b_{01}, b_{23}, b_{02}$, and $b_{13}$ are distinct. Hence we can find a vector $v$ with $Q_{0123}(v) \neq Q_{0231}(v)=0$. Since $Q_{0123}$ is not proportional to $Q_{0231}, \operatorname{dim} \mathcal{S}=2$.

The trace function is a nonzero linear form from $\mathcal{Q}$ to $\mathbb{R}$; its restriction to $\mathcal{S}$ is either nonzero, in which case $\operatorname{dim} \mathcal{T}=1$, or zero, in which event $\mathcal{T}=\mathcal{S}$ is twodimensional.

From (16) and 21) it follows that

$$
\operatorname{tr} Q_{i j k l}=b_{i j} \cdot b_{k l}=2\left(\lambda_{k i}-\lambda_{l i}\right)=2\left(\lambda_{l j}-\lambda_{k j}\right) .
$$

We note in passing that 222 and $(23)$ illustrate the meaning of equation (5): it simply says that the zero form $Q_{0123}+Q_{0231}+Q_{0312}$ has trace 0 . In addition, 23. implies that $\operatorname{tr} Q_{i j k l}=0$ is necessary and sufficient for the altitudes $h_{i}$ and $h_{j}$ to meet.

In particular, let us consider the quadratic form

$$
Q^{*}:=\lambda_{01} Q_{0123}+\lambda_{02} Q_{0231}+\lambda_{03} Q_{0312} .
$$

Equations 23 and the symmetry conditions 20 yield

$$
\operatorname{tr} Q^{*}=\lambda_{01}\left(2\left(\lambda_{02}-\lambda_{03}\right)\right)+\lambda_{02}\left(2\left(\lambda_{03}-\lambda_{01}\right)\right)+\lambda_{03}\left(2\left(\lambda_{01}-\lambda_{02}\right)\right)=0 .
$$

We are now in a position to state our main results: Theorem 7 states that the points on the altitudes satisfy the quadratic equation (26) if the Monge point $M$ is chosen as the origin. However, it remains open here whether or not $Q^{*}$ is identically zero. Thus equation 26 may be trivial $(0=0)$. We shall see later that this is the case if and only if the tetrahedron $T$ is orthocentric. Otherwise, $(26)$ is the equation of a (possibly degenerate) quadric carrying the four altitudes. This will be shown in Theorem 8 and the subsequent remarks.

Theorem 7. Let $P$ be a point on an altitude of a tetrahedron with Monge point $M$. Then $p:=\overrightarrow{M P}$ is a solution of the quadratic equation

$$
Q^{*}(x)=\left(\lambda_{01}-\lambda_{02}\right)\left(\lambda_{02}-\lambda_{03}\right)\left(\lambda_{03}-\lambda_{01}\right),
$$

where the quadratic form $Q^{*}$ and the scalars $\lambda_{i j}$ are given by 24) and 19), respectively.

Proof. If we apply a transposition on $(0,1,2,3)$, then both sides of 26 are multiplied by -1 , where we have to take into account the symmetry conditions $(20)$. So equation (26) remains unchanged, up to multiplication by \pm 1 , under any permutation of $(0,1,2,3)$. Therefore it is enough to establish that all $p$ determined by points $P$ on the altitude $h_{0}$ satisfy (26).

Formula 222 shows that

$$
Q^{*}=-\left(\lambda_{03}-\lambda_{01}\right) Q_{0123}+\left(\lambda_{02}-\lambda_{03}\right) Q_{0231} .
$$


Furthermore, (7) implies that $p \cdot b_{i j}=a_{0} \cdot b_{i j}=\lambda_{0 i}-\lambda_{0 j}$ whenever $i, j \neq 0$. Hence

$$
\begin{aligned}
Q^{*}(p) & =-\left(\lambda_{03}-\lambda_{01}\right)\left(p \cdot b_{01}\right)\left(p \cdot b_{23}\right)+\left(\lambda_{02}-\lambda_{03}\right)\left(p \cdot b_{02}\right)\left(p \cdot b_{31}\right) \\
& =-\left(\lambda_{03}-\lambda_{01}\right)\left(p \cdot b_{01}\right)\left(\lambda_{02}-\lambda_{03}\right)+\left(\lambda_{02}-\lambda_{03}\right)\left(p \cdot b_{02}\right)\left(\lambda_{03}-\lambda_{01}\right) \\
& =\left(p \cdot b_{12}\right)\left(\lambda_{02}-\lambda_{03}\right)\left(\lambda_{03}-\lambda_{01}\right) \\
& =\left(\lambda_{01}-\lambda_{02}\right)\left(\lambda_{02}-\lambda_{03}\right)\left(\lambda_{03}-\lambda_{01}\right),
\end{aligned}
$$

which completes the proof.

A hyperboloid is said to be equilateral if its asymptotic cone is equilateral. Recall that when $Q(x)=\rho$ is an equation of a hyperboloid, then $Q(x)=0$ is an equation of its asymptotic cone.

Theorem 8. If the four altitudes of a tetrahedron $T$ are mutually skew, then they are four generators of an equilateral hyperboloid $\mathcal{H}$. The Monge point $M$ is the center of $\mathcal{H}$.

Proof. Since the four altitudes are mutually skew, the scalars $\lambda_{01}, \lambda_{02}$, and $\lambda_{03}$ are distinct (see (21)). Accordingly, the quadratic form $Q^{*}$ assumes a nonzero value at each vertex of the tetrahedron, whence $Q^{*}$ cannot be the zero form. We already know from 25 that $\operatorname{tr} Q^{*}=0$ and from section 5 that $\operatorname{rank} Q^{*} \geq 2$. We claim that $\operatorname{rank} Q^{*}=3$.

Assume to the contrary that $\operatorname{rank} Q^{*}=2$. Then $Q^{*}(x)=0$ would define a pair of orthogonal planes and 26 would be the equation of a hyperbolic cylinder. However, the lines on a cylinder are parallel to each other, an absurdity.

Thus rank $Q^{*}=3$ and, since $Q^{*}(v)=Q^{*}(-v)$ for all $v$ in $\mathbb{E}$, equation (26) describes a quadric for which the Monge point is a center of symmetry, i.e., the reflection at the Monge point leaves the quadric surface invariant. Therefore it is an equilateral hyperboloid $\mathcal{H}$ whose asymptotic cone has equation $Q^{*}(x)=0$ and whose center is $M$. (It cannot be a hyperbolic paraboloid, because a paraboloid does not have a center of symmetry.)

Under the assumptions of Theorem 8 , we have $\operatorname{tr} Q_{i j k l} \neq 0$ for every choice of indices. In this case $\mathcal{T}=\mathbb{R} Q^{*}$ is a one-dimensional subspace of $\mathcal{Q}$. Also, since there are lines on the quadric surface $\mathcal{H}$, it is a hyperboloid of one sheet. The lines on such a quadric fall into two classes called reguli. Any two lines of the same regulus are skew, whereas each line $g$ of either regulus meets all lines but one of the other regulus; this exceptional line is parallel to $g$. Hence the four altitudes are four lines belonging to the same regulus. By Theorem 1. each orthocentric perpendicular has three points in common with $\mathcal{H}$, whence it is a line on this surface. This ensures that the four orthocentric perpendiculars belong to the other regulus, i.e., not to the one containing the altitudes. This is illustrated in Figure 10 however, only one orthocentric perpendicular is actually drawn. (On a hyperbolic paraboloid two distinct parallel lines do not exist. This demonstrates again that the quadric surface of the altitudes cannot be a hyperbolic paraboloid.) 


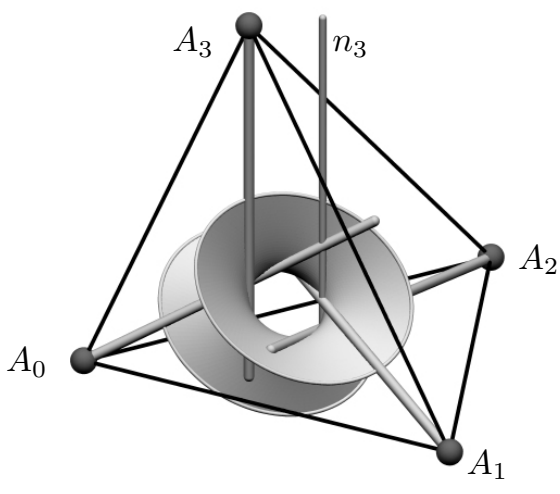

Figure 10.

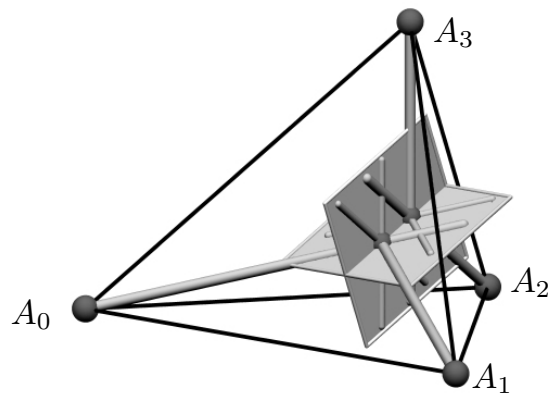

Figure 11 .

Next, we consider the case that two altitudes meet. Let, for example, $\lambda_{02}=\lambda_{03}$. Then (27) shows that (24) simplifies to

$$
Q^{*}=\left(\lambda_{01}-\lambda_{03}\right) Q_{0123} .
$$

If $\lambda_{01} \neq \lambda_{03}$, then $\operatorname{tr} Q_{0231} \neq 0$ and likewise $\operatorname{tr} Q_{0312} \neq 0$. We infer that $\mathcal{T}=\mathbb{R} Q^{*}=$ $\mathbb{R} Q_{0123}$ is one-dimensional. Also, there is no orthocenter, but the tetrahedron $T$ is semi-orthocentric. Furthermore, 26 is the equation of a degenerate quadric surface formed by the orthogonal midplanes $x \cdot b_{01}=0$ and $x \cdot b_{23}=0$. The first plane carries the altitudes $h_{2}$ and $h_{3}$ and the orthocentric perpendiculars $n_{2}$ and $n_{3}$, whereas the second plane carries the lines $h_{0}, h_{1}, n_{0}$, and $n_{1}$. The Monge point is the midpoint of the intersection points $h_{0} \cap h_{1}=n_{2} \cap n_{3}$ and $h_{2} \cap h_{3}=n_{0} \cap n_{1}$. The mutual position of these lines is portrayed in Figure 11 .

Finally, suppose that all altitudes are concurrent. Therefore $\lambda_{01}=\lambda_{02}=\lambda_{03}$ and all quadratic forms $Q_{i j k l}$ are traceless. Then $\mathcal{T}=\mathcal{S}$ is two-dimensional. In light of 28), $Q^{*}$ is a trivial quadratic form that does not deserve any attention. It is easily seen that the solution set of $Q_{i j k l}(x)=0$ contains all four altitudes, whence every equation $Q(x)=0$, where $Q$ in $\mathcal{T}=\mathcal{S}$ is nonzero, includes all four altitudes in its solution locus.

7. FINAL REMARKS AND FURTHER READING. If we are given an equilateral hyperboloid $\mathcal{H}$ of one sheet then any plane that is perpendicular to some generator $g$ of $\mathcal{H}$ meets the surface along an equilateral hyperbola or, if it is a tangent plane, along two orthogonal generators. This fact follows easily from Theorem 5 and is depicted in Figure 12 .

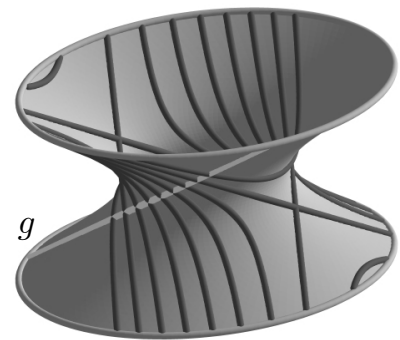

Figure 12 .

We remark that the hyperboloid of the altitudes (which is described in Theorem 8. meets each plane of the tetrahedron $T$ along an equilateral hyperbola. This is the 
result that a conic passing through a triangle and its orthocenter is an equilateral hyperbola 25, p. 172]. The quadric surface of the altitudes has a lot of further interesting properties (see [7).

Other recent publications dealing with the altitudes of a tetrahedron, the altitudes of simplexes in higher dimensional spaces, and other topics that are related to this circle of ideas are: [1, p. 371], 2], [3], [4], [5], [6, [7, 8], 9], 10, 11], 13], 14, [15, [16], [17, [18, [19], [21], [22, p. 376], [23.

\section{References}

[1] W.G. Boltjanski and I.M. Jaglom, Vektoren und ihre Anwendungen in der Geometrie, in Enzyklopädie der Elementarmathematik, P.S. Alexandroff, A.I. Markuschwitsch, and A.J. Chintschin, eds., vol. 4, VEB Dt. Verlag d. Wissenschaften, Berlin, 1969, pp. 295390.

[2] P. Couderc and A. Balliccioni, Premier Livre du Tétraèdre, Gauthier-Villars, Paris, 1935.

[3] N.A. Court, Notes on the orthocentric tetrahedron, Amer. Math. Monthly 41 (1934) 499-502.

[4] — Sur la géométrie du tétraèdre, Mathesis 51 (1937) 307-313.

[5] — On the theory of the tetrahedron, Bull. Amer. Math. Soc. 48 (1942) 583-589.

[6] - The biratio of the altitudes of a tetrahedron, Duke Math. J. 13 (1946) 383-386.

[7] — The tetrahedron and its altitudes, Scripta Math. 14 (1948) 85-97.

[8] — The semi-orthocentric tetrahedron, Amer. Math. Monthly 60 (1953) 306-310.

[9] — Modern Pure Solid Geometry, 2nd. ed., Chelsea, New York, 1964.

[10] R. Fritsch, «Höhenschnittpunkte» für $n$-Simplizes, Elemente Math. 31 (1976) 1-8. correction, ibid. 128.

[11] L. Gerber, The altitudes of a simplex are associated, Math. Mag. 46 (1973) 155-157.

[12] K.W. Gruenberg and A.J. Weir, Linear Geometry, Springer-Verlag, New York, 1977.

[13] S.R. Mandan, Altitudes of a simplex in four dimensional space, Bull. Calcutta Math. Soc. Suppl. 50 (1958) 8-20.

[14] - Semi-orthocentric and orthogonal simplexes in a 4-space, Bull. Calcutta Math. Soc. Suppl. 50 (1958) 21-29.

[15] — Altitudes of a general simplex in 4-space, Bull. Calcutta Math. Soc. Suppl. 50 (1958) 34-41.

[16] - Uni- and demi-orthocentric simplexes, J. Indian Math. Soc. 23 (1961) 169-184.

[17] — Altitudes of a simplex in n-space, J. Aust. Math. Soc. 2 (1962) 403-424.

[18] —, Uni- and demi-orthocentric simplexes. II., J. Indian Math. Soc. 26 (1962) 5-11.

[19] — Altitudes of a general n-simplex, J. Aust. Math. Soc. 5 (1965) 409-415.

[20] R. Mehmke, Ausdehnung einiger elementarer Sätze über das ebene Dreieck auf Räume von belibig vielen Dimensionen, Arch. Math. Phys. 70 (1884) 210-218.

[21] M. Miculita, On a property of the anticentre of a tetrahedron, Gaz. Mat., Bucur. 93 (1988) 100-102 (Romanian).

[22] B.A. Rosenfeld and I.M. Jaglom, Mehrdimensionale Räume, in Enzyklopädie der Elementarmathematik, P.S. Alexandroff, A.I. Markuschwitsch, and A.J. Chintschin, eds., vol. 5, VEB Dt. Verlag d. Wissenschaften, Berlin, 1969, pp. 337-383. 
[23] K. Satyanarayana, The tetrahedron the feet of whose altitudes are coplanar, Math. Stud. 50 (1982) 275-281.

[24] H. Schröter, Theorie der Oberflächen zweiter Ordnung und der Raumkurven dritter Ordnung als Erzeugnisse projektivistischer Gebilde, B.G. Teubner, Leipzig, 1880.

[25] J.G. Semple and G.T. Kneebone, Algebraic Projective Geometry, Oxford University Press, Oxford, 1998 (reprint of 1st. ed., 1952).

[26] M. Zacharias, Elementargeometrie und elementare nicht-euklidische Geometrie in synthetischer Behandlung, in Enzyklopädie der Mathematischen Wissenschaften III 1.2, W.Fr. Meyer and H. Mohrmann, eds., B.G. Teubner, Leipzig, 1914-1931, pp. 859-1172.

Hans Havlicek

Institut für Geometrie, Technische Universität, Wiedner Hauptstraße 8-10, A-1040

Wien, Austria

havlicek@geometrie.tuwien.ac.at

Gunter Weiss

Institut für Geometrie, Technische Universität, Zellescher Weg 12-14, D-01062 Dresden, Germany

weiss@math.tu-dresden.de 